\section{Artificial corneas}

Using transformed cell lines derived from the three main layers of human corneas, researchers in Canada and the US have constructed artificial corneas in vitro that appear to mimic the natural structure found in the eye. The work, reported in the 10 December issue of Science $(286,2169-2172,1999)$ suggests that corneal equivalents could be used in the near future to replace animal tests for eye irritation. To mimic the structure of the cornea, the researchers derived transformed cell lines from the epithelium, stroma, and endothelium of donated human corneas, and then cultured the appropriate cell layers on a polymer matrix. The resulting structures mimic natural corneas in architecture and transparency, and gene expression profiling shows that their response to irritants is also similar. Though replacing animal tests for eye irritants is a promising application, "the use of the cornea for transplants is the next phase of our project," says May Griffith, a researcher in the department of cellular and molecular medicine at the University of Ottawa (Ottawa, Ontario, Canada) and first author on the study. Procter and Gamble (Cincinnati, $\mathrm{OH}$ ) plans to commercialize the approach.

Gene sequencers attained several landmarks in recent weeks. Following the sequencing of the billionth base of the human genome (it was a guanine) by the Human Genome Project, scientists from the Sanger Center (Cambridge, UK), University of Oklahoma (Norman, OK), and Keio University School of Medicine in Tokyo reported on December 2 the first "almost continuous" sequence of the coding region of a human chromosomethat of chromosome 22 (Nature 402, 489-495, 1999). Although 545 genes were putatively identified (150 of which have homologs in other organisms), 11 sections of the $q$ region of the chromosome remain unsequenced because they were either impossible to clone or resistant to current sequencing techniques. At least 27 disorders have been linked to chromosome 22 . In November, scientists at the Institute for Genomic Research (Rockville, MD) also reported the full genome sequence of Deinococcus radiodurans, the most radiation-resistant bacteria currently known (Science 286, 1571-1577, 1999). Publication of the 180 million bp genome of Drosophila melanogaster genome, sequenced by Celera Genomics (Rockville, MD), is expected at the beginning of this year.

Research News Briefs written by Alan Dove, Robert Frederickson, and John Hodgson.

\title{
Illuminating applications for transgenic frogs
}

In an important proof-of-concept study, scientists at the Carnegie Institution of Washington (Baltimore, MD) have shown that a technique used to produce transgenic Xenopus laevis larvae for studies of early development can also be used to establish stable transgenic lines of the frogs (Proc. Natl. Acad. Sci. USA 96, 14389-14393, 1999). The finding paves the way for a wide range of experiments on later developmental stages and could also be useful in designing new bioassays for environmental monitoring.

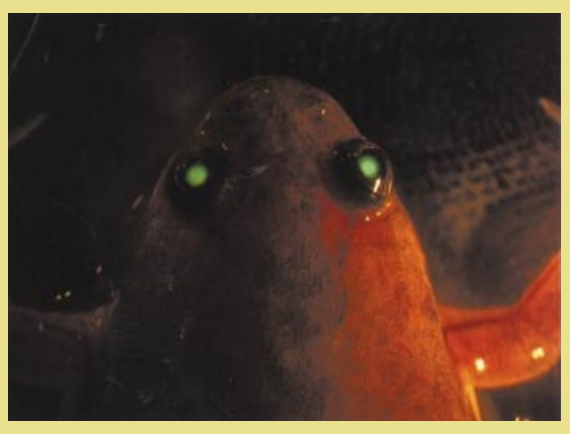
Previous work had shown that DNA can be integrated into demembranated Xenopus sperm nuclei by a restriction enzyme-mediated process. The modified nuclei can then be implanted into unfertilized frog eggs and the effects of the modification studied in the resulting embryos. As this approach results in wide variation in gene expression levels from one embryo to the next, the Carnegie scientists attempted to establish stable transgenic lines. By placing green fluorescent protein (GFP) in front of a variety of promoters and establishing independent frog lines, the team demonstrates that the transgenic frogs show appropriate gene expression patterns and are amenable to standard genetic experiments. In addition to basic science, transgenic Xenopus lines could be used for highly sensitive environmental testing. Donald Brown, senior author on the study, envisions a system in which "you just put the tadpoles in the water and they light up with GFP if there's [a bioactive contaminant] in the water."

\section{Bacteria with a silver lining}

Swedish scientists have reported the synthesis of silver crystals by a bacterial strain, Pseudomonas stutzeri, originally isolated from a silver mine (Proc. Natl. Acad. Sci. 96, 13611-13614, 1999). Silver is usually very toxic to microbes and has even been used as an antimicrobial agent. Although metalresistant microbes have been found before, the mechanism of resistance more commonly involves actively pumping the toxic ions out of the cell. These workers, led by Tanya Klaus, found that by culturing the bacteria in the presence of high concentrations of silver salts, they could create crystals of well-defined compositions and shapes in the region surrounding the plasma membrane. The size of the crystals identified by these workers (up to $200 \mathrm{~nm}$ ) suggests that they might eventually be used for the formation of thin metal films to absorb solar energy or to construct nanometer scale materials with unique optical and electrical properties useful in microelectronic applications. More traditional approaches to nanoscale synthesis tend to be inefficient and produce toxic by-products and the Pseudomonas system could represent a useful alternative. Klaus cautions, however, that the mechanism of the crystal formation needs to be better understood, before any practical application of the bacteria could be envisaged.

\section{Getting down to life's essentials}

Having sequenced the complete genomes of several organisms, scientists at the Institute for Genomic Research (TIGR; Rockville, MD) are now trying to determine the minimal set of genes required for life (Nat. Biotechnol. 17, 207, 1999). New results (Science 286, 2165-2169, 1999) from genome-wide transposon mutagenesis of Mycobacterium genitalium, which has the smallest known genome of a self-replicating organism, mark an important milestone for the work. The researchers used transposons to mutagenize the genomes of $M$. genitalium and its close relative, $M$. pneumoniae, then sequenced through the junctions of the transposon insertions to determine their locations. A total of 1354 distinct insertion sites within coding sequences were nonlethal, indicating that only 265-350 of the 480 protein-coding genes of $M$. genitalium, including 100 genes of unknown function, are essential for growth under laboratory conditions. To extend the research, the scientists are now hoping to develop a cassettebased artificial chromosome to determine the exact requirements for a minimal genome, but this work is still pending ethical review. An accompanying article discussing the bioethics of the project states that "the prospect of constructing minimal and new genomes does not violate any fundamental moral precepts or boundaries." 